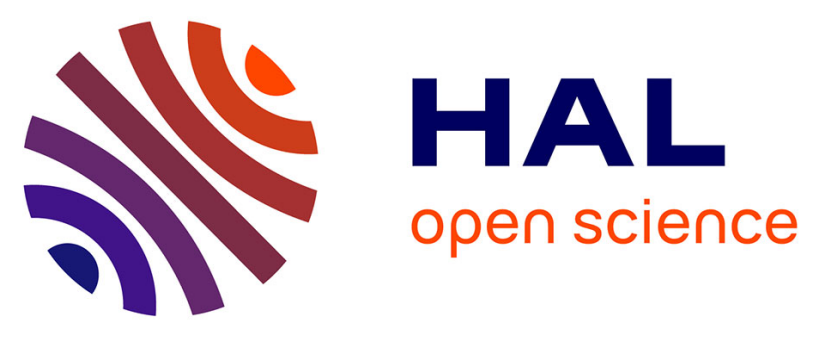

\title{
Periodic Mesoporous Organosilicas from Polyion Complex Micelles - Effect of Organic Bridge on Nanostructure
}

Albane Birault, Emilie Molina, Philippe Trens, Didier Cot, Guillaume Toquer, Nathalie Marcotte, Carole Carcel, John R Bartlett, Corine Gerardin, Michel Wong Chi Man

\section{To cite this version:}

Albane Birault, Emilie Molina, Philippe Trens, Didier Cot, Guillaume Toquer, et al.. Periodic Mesoporous Organosilicas from Polyion Complex Micelles - Effect of Organic Bridge on Nanostructure. European Journal of Inorganic Chemistry, 2019, 2019 (27), pp.3157-3164. 10.1002/ejic.201900487 . hal-03100976

\section{HAL Id: hal-03100976 https://hal.science/hal-03100976}

Submitted on 6 Jan 2021

HAL is a multi-disciplinary open access archive for the deposit and dissemination of scientific research documents, whether they are published or not. The documents may come from teaching and research institutions in France or abroad, or from public or private research centers.
L'archive ouverte pluridisciplinaire HAL, est destinée au dépôt et à la diffusion de documents scientifiques de niveau recherche, publiés ou non, émanant des établissements d'enseignement et de recherche français ou étrangers, des laboratoires publics ou privés. 


\title{
Periodic Mesoporous Organosilicas from Polyion Complex Micelles - Effect of Organic Bridge on Nanostructure
}

\author{
Albane Birault, ${ }^{[\mathrm{a}]}$ Emilie Molina, ${ }^{[\mathrm{a}]}$ Philippe Trens, ${ }^{[\mathrm{a}]}$ Didier Cot,${ }^{[\mathrm{b}]}$ Guillaume Toquer, ${ }^{[\mathrm{c}]}$ \\ Nathalie Marcotte, ${ }^{[\mathrm{a}]}$ Carole Carcel, ${ }^{[\mathrm{a}]}$ John R. Bartlett, ${ }^{[\mathrm{d}, \mathrm{e}]}$ Corine Gerardin, ${ }^{[\mathrm{a}]}$ and \\ Michel Wong Chi Man*[a] \\ [a] Institut Charles Gerhardt Montpellier - UMR 5253, CNRS, ENSCM, Univ. Montpellier, \\ Montpellier, France - E-mail: michel.wong-chi-man@enscm.fr - https://www.icgm.fr/michel- \\ wong-chi-man \\ [b] Institut Européen des Membrane - UMR 5635, ENSCM, CNRS, Univ. Montpellier, \\ Montpellier, France \\ [c] Institut de Chimie Séparative de Marcoule - UMR 5257, CEA, CNRS, ENSCM, Univ. \\ Montpellier, Marcoule, France \\ [d] University of the Sunshine Coast, 90 Sippy Downs Drive, Sippy Downs QLD 4556, Australia \\ [e] Western Sydney University, Locked Bag 1797 Penrith, NSW, 2751, Australia
}

\begin{abstract}
A new family of polyion complex (PIC)-based periodic mesoporous organosilicas, PICPMOs, obtained by the hydrolysis- condensation of organosilanes containing organic bridging units (phenylene, ethenylene and ethylene) in the presence of polyion complex (PIC) micelles as structure-directing agents (SDAs), is described. The electrostatic interactions between the acrylic acid functions of a poly(ethylene oxide)-bpoly(acrylic acid) doublehydrophilic block copolymer (DHBC) and the primary amine functions of a polyamine micellization agent control the formation of the core of the micellar complex, while the PEO chains of the micelle corona mediate the formation of the organic/inorganic interface that controls the evolution of the material. Herein, the micellization agent employed is an antibiotic drug, neomycin B. An important feature of this procedure is that the processing involves a "onepot" reaction, enabling the formation of the material and the direct encapsulation of the antibacterial agent. This results in a reduction in the number and cost of processing steps, while offering the opportunity to easily tune the hybrid mesostructure. The PICPMO materials obtained are organized on different length scales, from long-range-ordered 2D hexagonal structuring in the case of the phenylene bridge to weakly organized wormlike structures in the case of the ethylene unit. The effect of organic bridge structure on the nanostructure and physical properties of the PICPMO is discussed.
\end{abstract}

\section{Introduction}

Since the first reports of their preparation in the early 1990s, bridged silsesquioxanes, which consist of an organic group connected to at least two trialkoxysilyl groups, ${ }^{[1,2]}$ are now routinely used to prepare hybrid silicas for various applications including catalysis, ${ }^{[3]}$ photovoltaics, ${ }^{[4]}$ and nanomedicine. ${ }^{[5]}$ Around the same time, the first syntheses of purely silicabased periodic mesoporous materials using soft templating surfactants as structuring agents were reported. ${ }^{[6-8]}$ Soon after, this approach was extended to the preparation of mesoporous hybrid silica by mixing a monofunctional organosilane with a silica source such as TEOS. Vinyltrialkoxysilane was co-condensed with TEOS in the presence of CTAB cetyltrimethylammonium bromide) to anchor pendant vinyl groups within the generated periodic mesoporous hybrid silica; this approach was especially interesting since the material could be post-functionalized by bromination of the reactive vinyl bonds. ${ }^{9]}$ More recently, bridged organosilane precursors were used to produce a particularly interesting family of bridged silsesquioxanes referred to as periodic mesoporous organosilicas (PMOs). Similarly to 
the aforementioned mesoporous silica-based materials, PMOs also exhibit periodicity and uniformity of the pores within the hybrid framework. ${ }^{\left[{ }^{10-12]}\right.}$ This approach offers the possibility of having the organic functions distributed uniformly within the walls of the pores ${ }^{[13]}$ and, in addition, facilitates the design of multifunctional hybrid materials such as PMO nanoparticles which can be used in catalysis, adsorption, drug delivery, sensing, etc. ${ }^{[14]}$ Although the development, characterization and uses of PMOs have been reviewed by several authors, ${ }^{[15-18]}$ many additional applications can be anticipated, based on the possibility of mixing and localizing the functionalities in the pores or in the walls, controlling the proximities of multiple organic functions, ${ }^{[5,19]}$ improving the wall crystallinity, ${ }^{[20]}$ as well as controlling the pore size ${ }^{[21-}$ ${ }^{25]}$ and the wall thickness of the resulting materials. However, a limitation of traditional surfactant-based soft templates is the relatively small size of the pores that are typically obtained, which limits the potential applications of the resulting PMOs.

Block copolymers have already been employed to synthesize large pore PMOs. ${ }^{[26,27]} \mathrm{A}$ decade ago, it was shown that double hydrophilic block copolymers (DHBCs), when combined with oppositely charged polyelectrolytes, can form polyion complex (PIC) micelles which can direct the pore structuring of silica. In addition, owing to the reversible self-assembly of the PIC system it has also been demonstrated that the templating PIC polymers can be easily recovered and re-used. ${ }^{[28]}$ Poly(ethylene oxide)- $b$-poly(acrylic acid) (PEO- $b$-PAA) doublehydrophilic block copolymers associated with an oligochitosan $[\mathrm{OC}]^{[29]}$ as the micellization agents were used as efficient templates of ordered mesoporous silica materials with tunable mesostructures. Subsequently, newly synthesized mesoporous silicas were formed using aminoglycoside antibiotics as micellizing agent, thus affording mesostructured materials directly loaded with the drug. ${ }^{[30]}$ We recently extended the use of such systems to synthesize PMOs from phenylene-bridged organosilane (BTEB: 1,4-bis(triethoxysilyl)benzene) using water as solvent. The PICPMO obtained with a PEO- $b$-PAA/OC system was a lamellar bridged silsesquioxane with a large interlamellar distance of around $15 \mathrm{~nm} .{ }^{[31]}$ By using neomycin B (NM) as a polyamine to form PIC micelles with the DHBC in a solvent mixture of $\mathrm{H}_{2} \mathrm{O} / n$-propanol, 2D hexagonal PICPMOs could be obtained, and their application as an autonomous drug delivery system was demonstrated. A key feature of this approach is the dual role of NM.The antibiotic polyamine not only serves as a structuring agent but also as a bactericide, which could be autonomously released within an acidic medium. ${ }^{[32]}$

In this study, the effects of the bridging unit on the mesostructure of the PICPMOs obtained are investigated. In particular, the materials obtained with ethylene and ethenylene bridging organics, which are well known to form PMOs, are compared to the results obtained using a previously reported phenylene bridge system ${ }^{[32]}$ to determine the influence of the bridging unit on the formation of a new family of mesoporous organosilicas.

\section{Results and Discussion}

The general approach used to synthesize PICPMO materials is illustrated in Scheme 1. PIC 
micelles are formed using PEO- $b$ - PAA as DHBC and NM as the polybase. BTEB, BTSE $(1,2-$ bis(triethoxysilyl)ethylene) or BTSEENE (1,2-bis(triethoxysilyl)ethenylene) are used as the organosilylated precursor. In a typical procedure, NM was mixed with PEO- $b$-PAA in water at room temperature. The solution $\mathrm{pH}$ was then reduced to about 2 by addition of an appropriate amount of $3 \mathrm{M} \mathrm{HCl}$ solution and the organosilylated precursor was added. After hydrolysis of the precursor, the solution $\mathrm{pH}$ was increased to 6.5 to induce both PAA-NM complexation and polycondensation of the organosilane species. A precipitate was recovered by centrifugation and dried to form an off-white powder. The structures of the as-prepared materials were evaluated by cross-sectional TEM and SEM (Figure 1), SWAXS (Figure 2) and $\mathrm{N}_{2}$ sorption analyses (Figure 3).

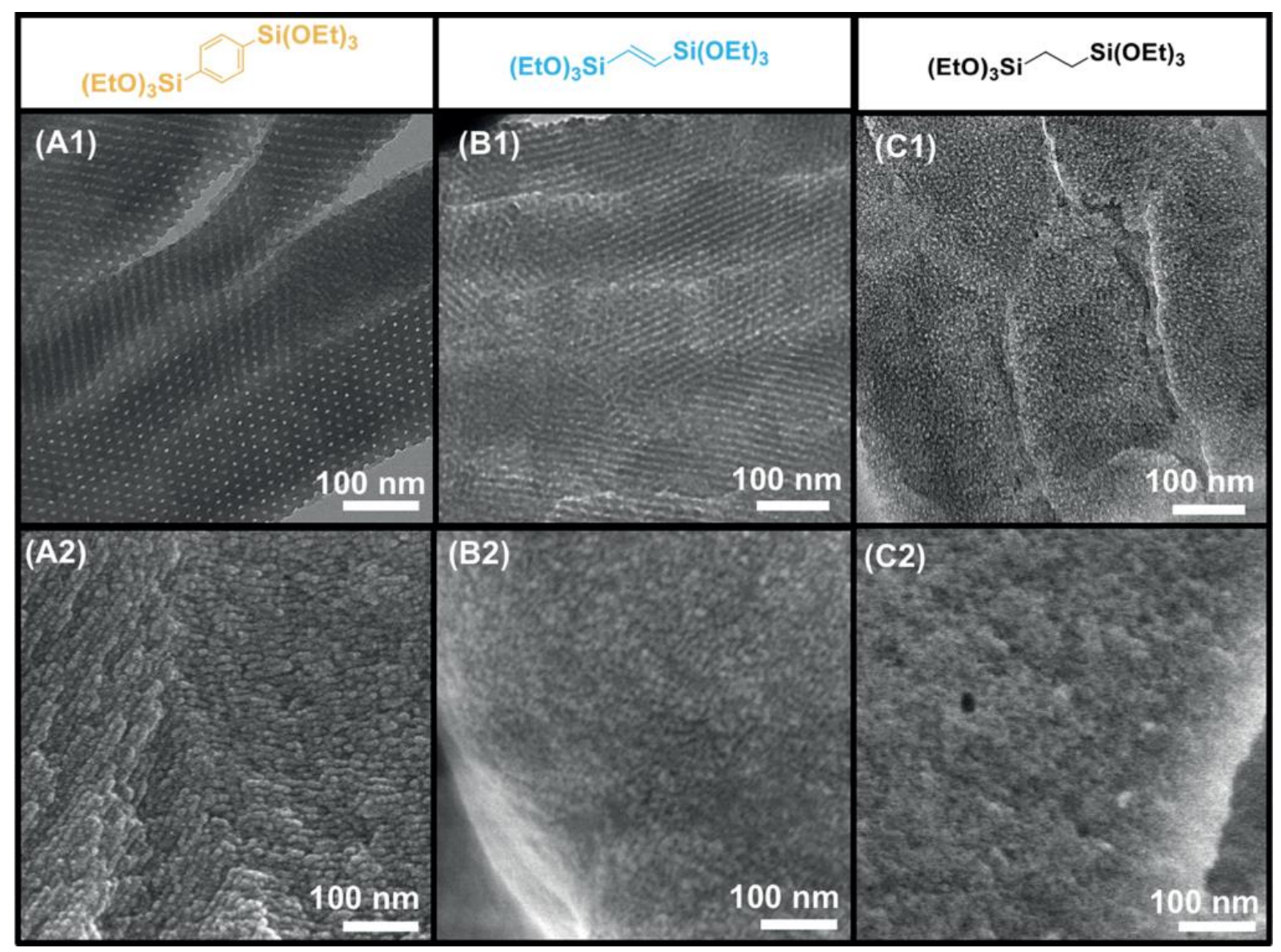

Figure 1. TEM (top row) and SEM (bottom row) micrographs of the BTEB-based PICPMO (A1, A2) BTSEENEbased PICPMO (B1, B2) and BTSE-based PICPMO (C1, C2).

The BTEB-based material, which has been described previously, ${ }^{[32]}$ exhibits a well-defined 2D hexagonal mesostructure, with excellent organization over distances of several hundred $\mathrm{nm}$ (Figure 1 A1). This is clearly shown from the SWAXS spectrum (Figure 2), which exhibits welldefined peaks at $0.45,0.79$ and $0.91 \mathrm{~nm}^{-1}$ (equivalent pseudo-Bragg spacings of 14,8 and $7 \mathrm{~nm}$, respectively). With the incorporation of the ethenylene bridging group in place of the phenylene group, the as-synthesized BTSEENE-based PICPMO also exhibits an organized hexagonal-type mesophase (Figure 1 B1). The TEM image reveals some less-organized wormlike domains that consequently reduce the length scale of ordering, as also observed via SWAXS (Figure 2). The scattering pattern of the as-prepared BTSEENE-based PICPMO exhibits two relatively broad peaks at low $q\left(0.56 \mathrm{~nm}^{-1} ; 0.96 \mathrm{~nm}^{-1}\right)$. These peaks are assigned to pseudo-Bragg reflections from the (100) and (110) planes ( $p 6 \mathrm{~mm}$ symmetry), consistent with 
the ordered 2D hexagonal mesostructure observed via TEM. However, these peaks are somewhat broader than those observed for the BTEB-based material, consistent with less longrange order in the BTSEENE-based product. It should be noted that BTSEENE exists as two isomers $(E$ and $Z)$ and that a mixture of 80:20 is commercially available. In our work, we synthesized an E/Z:99:1 mixture (Figure S1) which is known to induce better structuring of the hybrid material, as previously reported. ${ }^{[22]}$

The TEM micrograph of the PICPMO hybrid formed from BTSE (ethylene) reveals a porous material with mainly wormlike domains, consistent with a lack of long-range ordering within the material and in contrast with those observed for BTEB and BTSEENE (Figure 1 C1). The corresponding SEM image (Figure 1 C2) confirms this result, where a somewhat disordered morphology is clearly evident. These results are consistent with the single, very broad signal at $q$-values centered at $0.52 \mathrm{~nm}^{-1}$ in the SWAXS spectrum in Figure 2 (black diffractogram), reflecting the absence of long-range and well-defined organization. In particular, the pseudoBragg peak obtained from the $q$-values of $0.52 \mathrm{~nm}^{-1}$ corresponds to a characteristic distance of $12.0 \pm 0.8 \mathrm{~nm}$. From these studies, it is evident that BTSEENE PICPMO, although less organized than BTEB PICPMO, exhibits better structuring than its BTSE analogue. The very broad peak found for BTSE PICPMO compared with the SWAXS spectra of BTEB and BTSEENE PICPMOs is consistent with less

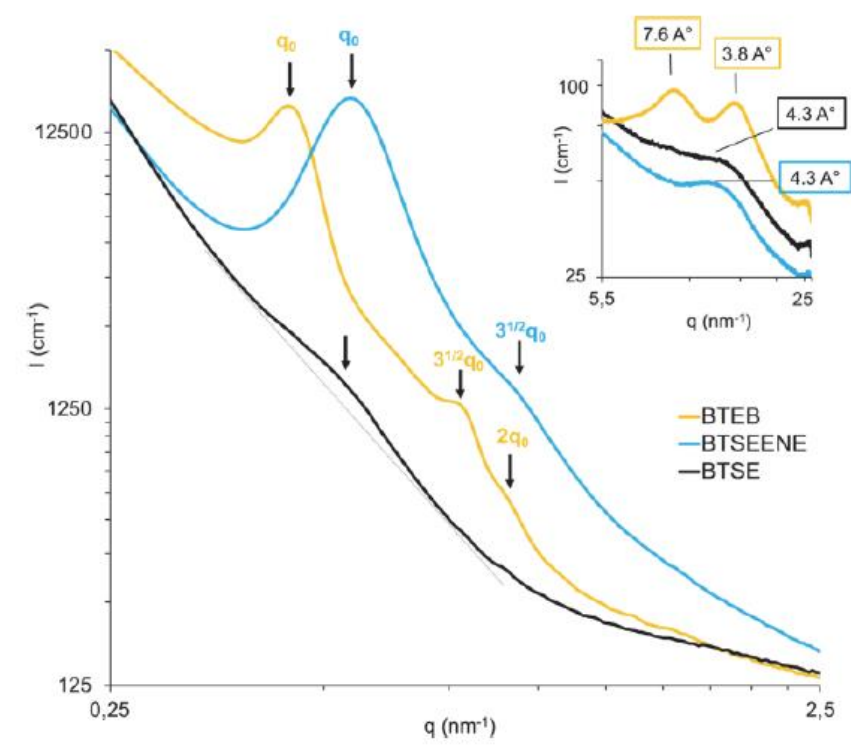

Figure 2. SWAXS profiles of the BTEB-based PICPMO, BTSEENE-based PICPMO and BTSE-based PICPMO. organization in the former hybrid silica.

Interestingly, as already reported, at high $q$-values the BTEB PICPMO profile exhibits two characteristic distances of 0.76 and $0.38 \mathrm{~nm}$, consistent with a molecular-scale periodicity. Indeed, it has been proposed that the distance of $0.76 \mathrm{~nm}$ corresponds to the spacing between the aryl moieties ${ }^{[20]}$ while $d=0.38 \mathrm{~nm}$ is assigned to the distance between two aromatic rings, which are ordered via $\pi-\pi$ interactions. Furthermore, the SWAXS profiles of BTSEENE and BTSE PICPMOs exhibit a specific distance at $d=0.43 \mathrm{~nm}$, reflecting a molecular-scale organization of the organic functions that can presumably be assigned to the repeat distance between adjacent organic bridges. It is noteworthy that the very broad peak in the case of BTSE PICPMO indicates a less crystalline organization.

The as-prepared PICPMOs were treated with $\mathrm{HCl}$ to dissociate the PIC micelles in acidic medium (due to PAA neutralization); this step is expected to remove NM from the material, ${ }^{[29]}$ and thus liberate the porosity within the PMOs. The resulting porosity was evaluated by performing nitrogen sorption at $77 \mathrm{~K}$ (Figure 3, Table 1). The texture of the BTSEbased PICPMO exhibits a Type II adsorption isotherm (Figure 3C) characteristic of nonporous materials. In contrast, nitrogen sorption performed on the BTSEENE- and BTEB-based samples (Figures 3A, 3B) reveals Type IV isotherms typical of mesoporous materials, characterized by a strong uptake at intermediate relative pressure and a flat saturation plateau once the mesopores are filled. A BJH analysis of the capillary condensation processes led to mean pore sizes of 3.4 and $8.0 \mathrm{~nm}$, respectively. The hysteresis loops which are observed at high relative pressure can be classically attributed to some interparticle porosity, generated by 
the aggregation of particles. The increase in pore size from BSEENE to BTEB is correlated with the extent of long-range ordering revealed by TEM, reflecting the complex series of equilibria that control the evolution of PICPMOs which involve $\mathrm{pH}$; interactions between the DHBC, NM and condensing PMO framework; protonation of the PMO framework; etc. The decrease in the extent of ordering when changing the precursor from BTEB to BTSEENE to BTSE suggests that the use of functional bridging groups that have the ability to interact through (for example) $\pi-\pi$ interactions, and thus play a structure-directing role on the molecular scale, favors the cooperative assembly of the hybrid system and the formation of long-rangeordered hybrid mesostructures between PIC micelles and organosilica species. In addition, the relative size/volumes of the pores in the BTEB- and BTSEENE-based materials would be expected to be influenced by the more hydrophilic character of BTSEENE compared with BTEB, presumably generating stronger interactions with the hydrophilic NM forming the core of the micelle in the former case, and as a direct consequence, reducing the size of the mesopores.

(A)

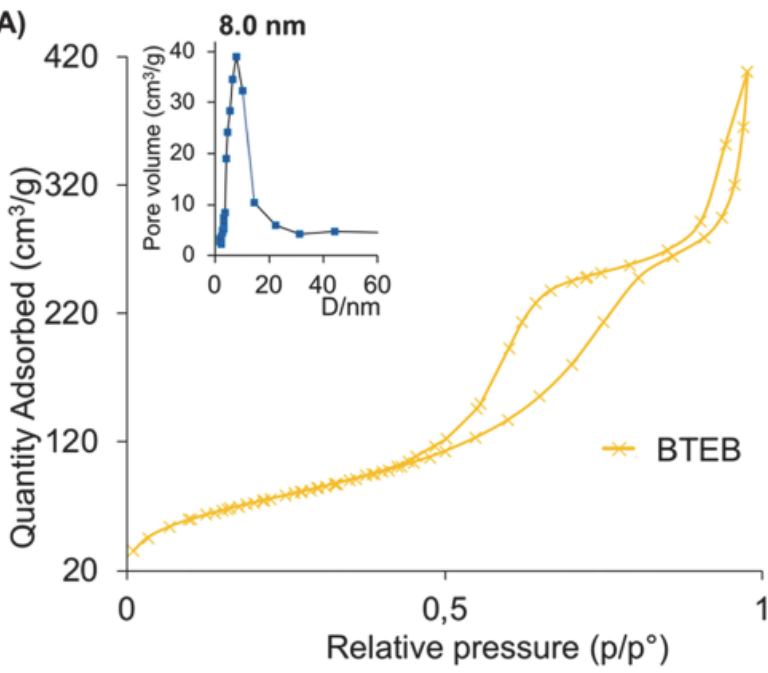

(C)
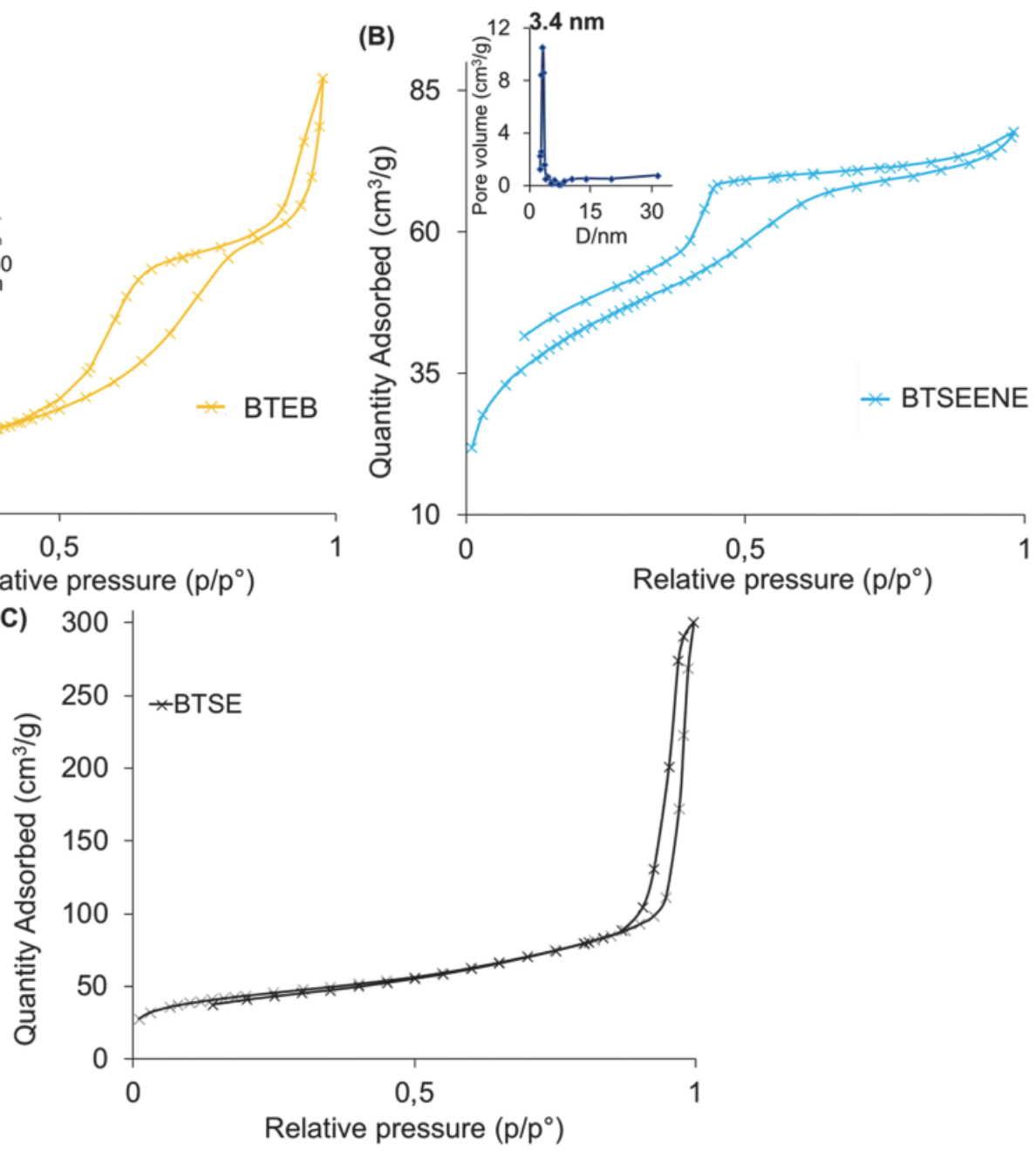

Figure 3. $\mathrm{N}_{2}$ sorption analysis of PICPMO prepared from (A) BTEB, (B) BTSEENE and (C) BTSE precursor.

Table 1. $\mathrm{N}_{2}$ sorption analysis of PICPMOs.

\begin{tabular}{llllll}
\hline Precursor & $\begin{array}{l}\text { BET Surface Area } \\
{\left[\mathrm{m}^{2} \mathrm{~g}^{-1}\right]}\end{array}$ & $\begin{array}{l}\text { BJH Pore Volume } \\
{\left[\mathrm{cm}^{3} \mathrm{~g} \mathrm{~g}^{-1}\right]}\end{array}$ & BJH Pore Diameter $[\mathrm{nm}]$ & Wall Thickness [nm] & $\begin{array}{l}\mathrm{t} \text {-Plot Micropore Volume } \\
{\left[\mathrm{cm}^{3} \mathrm{~g}^{-1}\right]}\end{array}$ \\
\hline BTSE & 148 & 0.46 & - & - & $1.8 \times 10^{-2}$ \\
BTSEENE & 148 & 0.11 & 3.2 & 7.6 & $6.4 \times 10^{-3}$ \\
BTEB & 270 & 0.63 & 8.0 & 8.2 & \\
\hline
\end{tabular}


The non-closure of the sorption isotherm in the case of the BTSEENE-derived material, which is typically observed for this particular system, is noteworthy, and provides additional insights into the molecular-level structure of the material. Similar non-closure has been previously reported for polymer-based systems ${ }^{[33,34]}$ and presumably arises in our system due to the presence of a significant abundance of PEO polymer segments within the mesopores of the BTSEENE-derived material. More specifically in our case, the delayed desorption of nitrogen may be interpreted as follows. Condensation of nitrogen within the pores is exothermic $\left(\Delta \mathrm{vap} H \approx-5.6 \mathrm{~kJ} / \mathrm{mol}^{[35,36]}\right)$, and the energy released upon adsorption enables the polymer chains to flex and accommodate nitrogen within microporous domains. However, during the endothermic desorption process, the polymer chains remain rigid, trapping nitrogen within these domains, thus preventing the desorption of nitrogen as the partial pressure is reduced. Llewellyn et al. studied the calcination of MCM-41 mesophases, ${ }^{[37]}$ and found that the desorption branches of the sorption isotherm closed the adsorption branches only in the case of samples calcined at high temperatures, due to the influence of residual organic species within the pores. Similar findings were reported by Boutin et al. on silylated MCM-41 materials, in which they investigated the influence of grafted organic chains on nitrogen adsorption isotherms. ${ }^{[38]}$ Additionally, it can be noted that the branches of the hysteresis loop in the case of the BTSEENE-derived sample are not parallel, which is attributed to so-called "catastrophic desorption" of nitrogen from small mesopores at $\mathrm{p} / \mathrm{p}^{\circ}=0.42$. The speciation of the acid-treated PICPMO frameworks was assessed by ${ }^{13} \mathrm{C}$ and ${ }^{29} \mathrm{Si}$ solid-state NMR measurements (Figure 4).
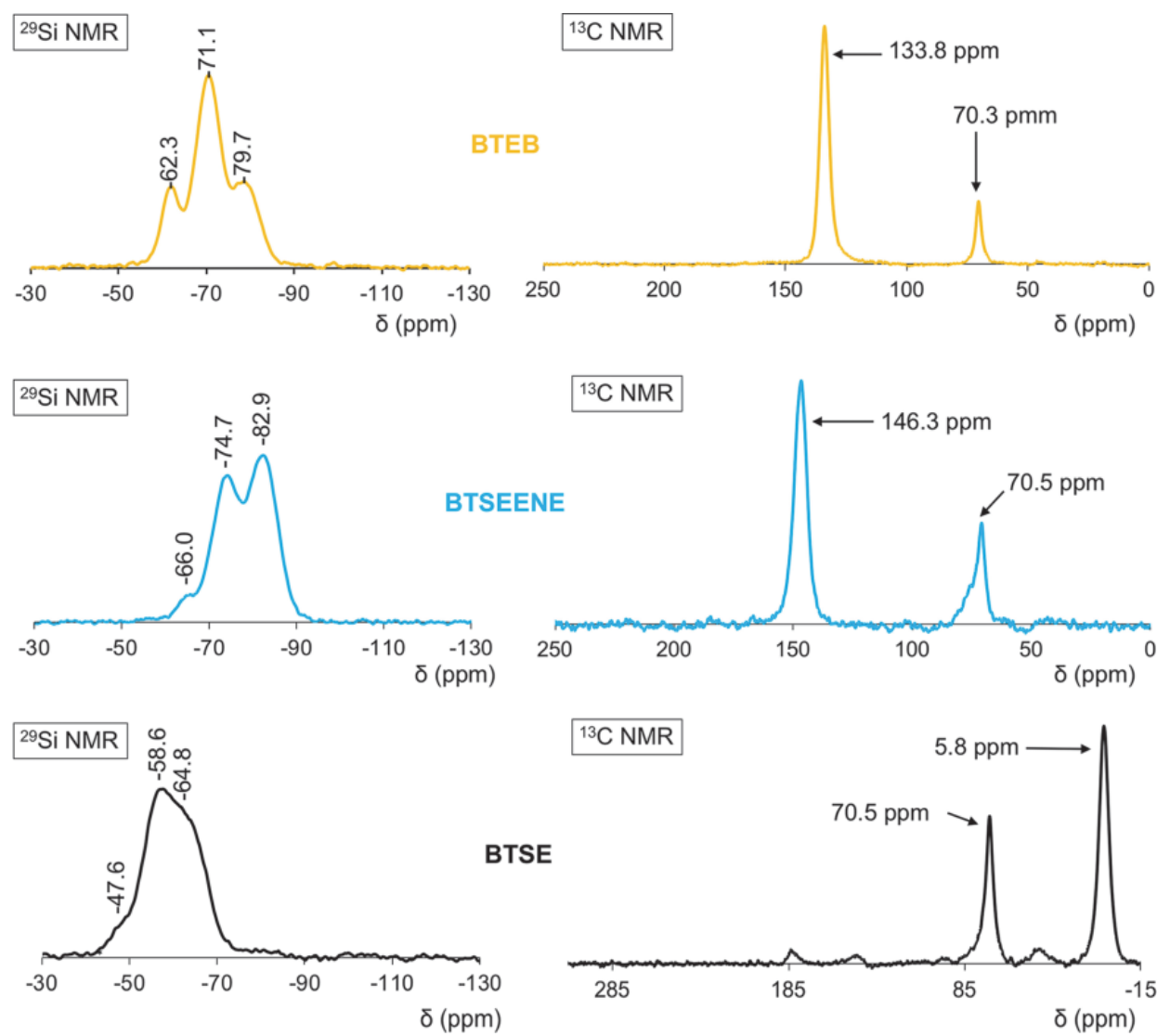

Figure 4. Solid state ${ }^{13} \mathrm{C}$ and ${ }^{29} \mathrm{Si}$ CPMAS NMR spectra of BTEB-, BTSEENE- and BTSE-based PICPMOs.

The ${ }^{13} \mathrm{C}$ CPMAS NMR spectrum obtained from BTSEENE PICPMO (Figure 4 blue line) exhibits a sharp signal at $146.7 \mathrm{ppm}$, characteristic of the $\mathrm{Csp}^{2}$ site of the $E$-isomer ethenylene bridging functions within the hybrid matrix. ${ }^{[39]}$ The BTEB PICPMO spectrum (yellow line) shows a peak at $133.8 \mathrm{ppm}$ corresponding to the phenyl carbon atoms, while that 
of the BTSE PICPMO (black line) exhibits a peak at $5.8 \mathrm{ppm}$ corresponding to the $\mathrm{Csp}^{3}$ site in the bridged ethylene silsesquioxane matrix. Moreover, the peak at 70.3-70.5 ppm, which is present in all cases, is assigned to $-\mathrm{CH}_{2}$ groups of the PEO block of the DHBC, revealing the strong interaction of the neutral chains within the framework even after acid treatment. ${ }^{29} \mathrm{Si}$ CPMAS NMR spectra, also presented in Figure 4, highlight three resonances at $-66.0,-74.7$ and $-82.9 \mathrm{ppm}$ for the BTSEENE PICPMO that are attributed to $\mathrm{T}^{1}(\mathrm{CH}=\mathrm{CH}-\mathrm{Si}(\mathrm{OH}) 2(\mathrm{OSi}))$, $\mathrm{T}^{2}\left(\mathrm{CH}=\mathrm{CH}-\mathrm{Si}(\mathrm{OH})(\mathrm{OSi})_{2}\right)$ and $\mathrm{T}^{3}\left(\mathrm{CH}=\mathrm{CH}-\mathrm{Si}(\mathrm{OSi})_{3}\right)$ sites, respectively. In this particular case, the $\mathrm{T}^{3}$ units are more abundant than the $\mathrm{T}^{2}$ and $\mathrm{T}^{1}$ units, in contrast to the BTEB PICPMO spectrum where the $\mathrm{T}^{2}$ sites predominate. The ${ }^{29} \mathrm{Si}$ CPMAS NMR spectrum of the NM-free BTSE PICPMO exhibits three signals at $-47.6,-58.6$ and $-64.8 \mathrm{ppm}$ attributed, respectively, to $\mathrm{T}^{1}, \mathrm{~T}^{2}$ and $\mathrm{T}^{3}$ sites. The different chemical shifts observed in the latter case are attributed to the change of $\mathrm{C}$ hybridization from $\mathrm{sp}^{3}$ to $\mathrm{sp}^{2}$. Furthermore, a comparison of the ${ }^{29} \mathrm{Si}$ solid state NMR spectra of BTSE- and BTSEENE-based PICPMOs shows that the BTSEENE-based material exhibits more complete condensation than the BTSE-based material. The $\mathrm{C}=\mathrm{C}$ double bond covalently attached to the silicon atom in the BTSEENE-based system would be expected to render the silicon site more susceptible to nucleophilic attack than in the corresponding BTSE-based material, which would result in a greater abundance of $\mathrm{T}^{3}$ sites. In all cases, the incorporation and retention of DHBC in the material is verified by the distinct peak at around $70.3 \mathrm{ppm}$ in the ${ }^{13} \mathrm{CNMR}$ spectra. Furthermore, the absence of the specific peaks of the $\mathrm{NM}^{[32]}$ validates the elimination of the polyamine during acid washing. The absence of any peaks arising from $\mathrm{Q}^{\mathrm{n}}$ species at -90 to $-120 \mathrm{ppm}$ indicates that no $\mathrm{Si}-\mathrm{C}$ cleavage occurred during the acid treatment.

The mass fractions (wt.-\%) of DHBC, NM, organic bridging units, physisorbedchemisorbed $\mathrm{H}_{2} \mathrm{O}$ and key molar ratios, calculated from TGA and elemental analysis, are summarized in Table 2. The key parameter (N/AA) and (EO/Si) represent the molar ratio between polyamine and acrylic acid functions and between PEO chains and siliceous species, respectively. The composition of the BTEB-based PICPMO is characterized by two key molar ratios, N/AA (1.44) and EO/Si (1.33). These results confirm the presence of strong complexation between the NM and the DHBC via electrostatic interactions, together with the retention of significant quantities of the $\mathrm{DHBC}$ within the material.

Table 2. Chemical composition (wt \%) of BTEB-, BTSEENE- and BTSE-basedPICPMO materials prior to acid washing.

\begin{tabular}{lllll}
\hline & Precursor & BTEB & BTSEENE & BTSE \\
\hline \multirow{2}{*}{ As-prepared } & \% Walls & 40.7 & 58.7 & 61.6 \\
Materials & \% DHBC & 31.6 & 24.7 & 26.5 \\
Wt\% & \% Water & 13.2 & 7.1 & 4.5 \\
& 14.5 & 9.5 & 7.4 \\
\hline \multirow{2}{*}{ Molar ratio } & N/AA & 1.44 & 0.98 & 0.57 \\
& EO/Si & 1.33 & 0.74 & 0.76 \\
\hline
\end{tabular}

Similarly, the composition of the BTSEENE-based material indicates that significant quantities of NM are also retained within the siliceous framework (N/AA =0.98). However, the integration of PEO blocks within the material is significantly reduced compared to the phenylene-bridged materials $(\mathrm{EO} / \mathrm{Si}=0.74$ and 1.33 , respectively), which is consistent with the reduction in the extent of long-range ordering in the case of the BTSEENE-based material.

Finally, the relatively low quantities of both NM and DHBC retained within the BTSEbased PICPMO $(\mathrm{N} / \mathrm{AA}=0.57 ; \mathrm{EO} / \mathrm{Si}=0.76)$ are presumably responsible for the relatively short-range order observed in this material.

The apparent evolution from a well-structured BTEB PICPMO to a weakly structured BTSE PICPMO appears to occur when the rigidity and potentially favorable interactions between the bridging units decrease. In particular, we propose: 
- BTEB provides both rigidity and $\pi-\pi$ stacking interactions, which strongly favor molecularscale organization within the material; ${ }^{[20,21]}$

- BTSEENE has a semi-rigid organic bridging system, which still plays a nanostructuring role $;{ }^{[22,25]}$ while

- BTSE is much more flexible and is thus less likely to promote well-defined ordering of the structure.

Consequently, the extent of long-range structuring follows the trend phenylene-bridged PICPMO > ethenylene-bridged PICPMO > ethylene-bridged PICPMO.

Furthermore, solid-state NMR spectroscopy demonstrated that the different organic bridging functions are covalently incorporated within the framework and exhibit no degradation under the synthesis conditions used in this work. It is noteworthy that the extent of condensation is highly dependent on the nature of the organosilane bridging units, with BTSEENE and

BTSE PICPMO materials, in particular, exhibiting more extensive polycondensation compared to BTEB-derived PICPMO. This result highlights differences in the extent of the condensation reaction between the various hybrid materials, which might be related to (1) a difference in hydrolysis rates of the precursor organosilanes; and (2) an inhibition of the condensation reactions due to strong ordering and a lower flexibility of the organosilica framework induced by $\pi-\pi$ stacking organization. The incorporation of organic functions such as $\mathrm{C}=\mathrm{C}$ double bonds provides an opportunity to post-functionalize the pore walls of the PICPMOs materials using simple and efficient chemical reactions. For example, it is well-known that carbon-carbon double bonds readily react with $\mathrm{Br}_{2}$ vapor, as previously demonstrated in PMO systems, ${ }^{[9]}$ via a radical reaction mechanism, owing to the facile photolytic cleavage of $\mathrm{Br}_{2}{ }^{\left[{ }^{40]}\right.}$ Figure 5 illustrates the ${ }^{13} \mathrm{C}$ solid state CPMAS NMR spectrum of BTSEENE PICPMO before (blue line) and after exposure to gaseous $\mathrm{Br}_{2}$ for $24 \mathrm{~h}$ (dashed-black line). The spectrum of the brominated PICPMO hybrid clearly exhibits an intense peak at $32.5 \mathrm{ppm}$, which is assigned to the formation of $\mathrm{C}$-Br species during the bromination reaction. However, the bromination of the double bonds was not complete, as revealed by the ${ }^{13} \mathrm{C}$ NMR spectrum (dashed-black line) with remaining $\mathrm{C}=\mathrm{C}$ units revealed by the peak at around $150 \mathrm{ppm}$. A semi-quantitative analysis of the extent of bromination using thermogravimetric analysis (ESI, Figures S4 and S5) indicates that around $11 \%$ of the $-\mathrm{CH}=\mathrm{CH}-$ bridges were brominated under these conditions. In addition, the absence of significant $\mathrm{Si}-\mathrm{C}$ bond degradation by potential attack of the bromine on electrophilic silicon sites was confirmed by the absence of $\mathrm{Q}^{\mathrm{n}}$ species (Figure $\mathrm{S} 3$ ).
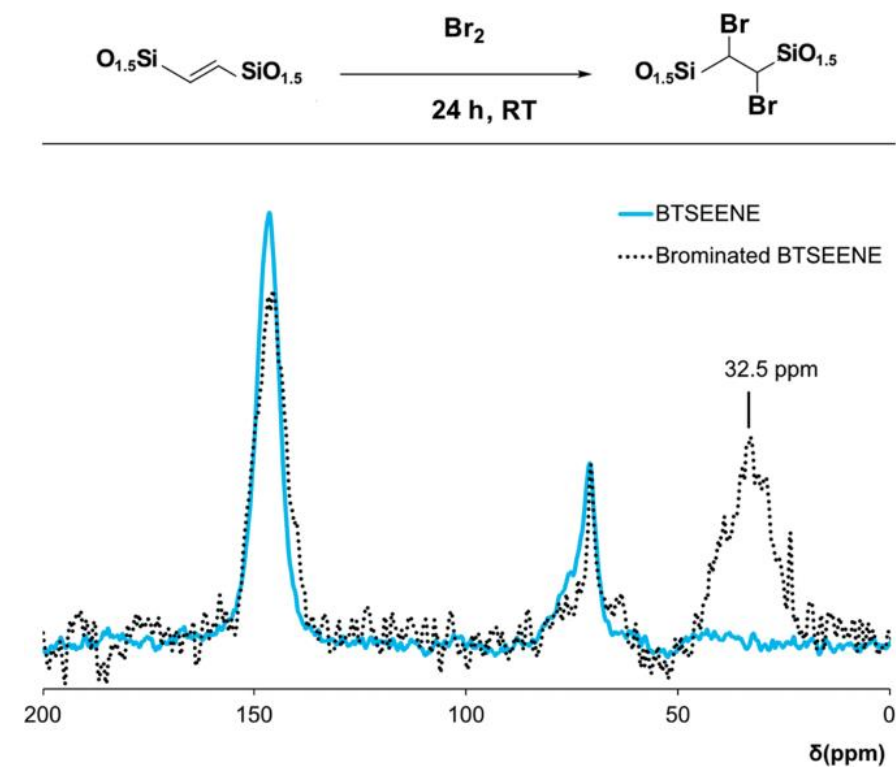

Figure $5 .{ }^{13} \mathrm{C}$ solid state CPMAS NMR spectra of the BTSEENE-based and the brominated BTSEENE-based PICPMOs. 
The post-functionalization of BTSEENE-derived PICPMO was also characterized by EDX-HRTEM as shown in Figure 6. Elemental mapping of brominated BTSEENE PICPMO reveals the uni- form distribution of bromine atoms in the material (brown color). It is notable that the carbon atoms (red color) are also homogeneously distributed within the PMO shell, consistent with the covalent bonding of the 1,2-dibromoethylene bridging groups generated after bromination.

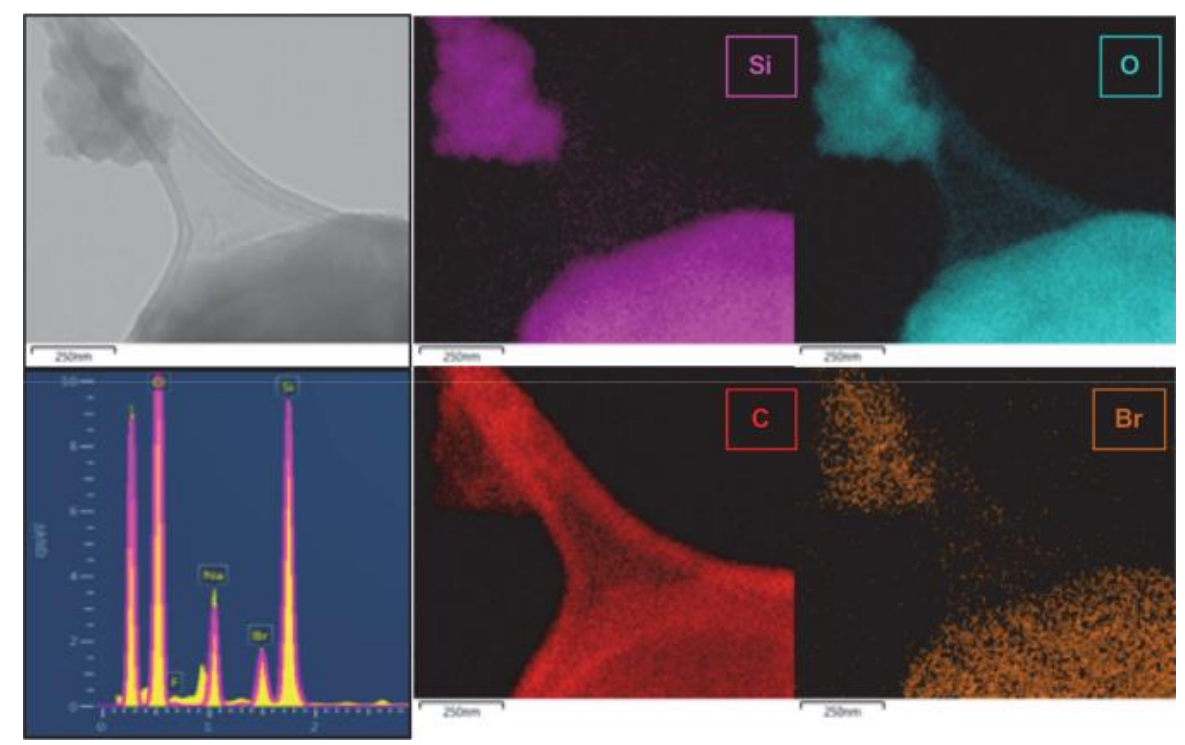

Figure 6. EDX-HRTEM analysis of the brominated BTSEENE-based PICPMO.

This preliminary study shows that PICPMO can be successfully post-functionalized by bromination via a low-cost and efficient procedure under mild condition. This opens the way to the synthesis of multi-hybridized advanced materials, with a wide range of potential applications.

\section{Conclusions}

A versatile synthetic strategy for producing PICPMO hybrids using several types of organo-bridged bis-silanes has been demonstrated. The aim of this study was to demonstrate the feasibility of synthesizing PICPMOs with different bridging units, including ethenylene (BTSEENE), ethylene (BTSE) and phenylene (BTEB) organic functions. It is demonstrated that PICPMO hybrids with a defined mesostructure and intrinsic functional flexibility could be synthesized with BTEB and BTSEENE while the saturated-carbon precursor BTSE led to a nanostructured hybrid material exhibiting a wormhole pore structure.

Interestingly it was shown that the organic bridging groups can be further modified by post-treatment reactions within the solid materials while maintaining the ordered structure. In particular, the ethenylene bridging groups of PICPMO were functionalized by bromination to form brominated $\mathrm{Csp}^{3}$ covalently bonded to the hybrid silica matrix, as demonstrated by ${ }^{13} \mathrm{C}$ solid state NMR studies. The possibility of functionalizing such PICPMOs by post-synthesis will pave the way to new functional hybrid silicas for many applications. For example, the inclusion of bridging organics incorporating disulfide moieties offers the possibility of preparing (bio)degradable systems for nanomedicine, in addition to applications exploiting the resulting thiol species for complexing metallic nanoparticles, complexation of heavy metals, post-functionalization of the thiols via click chemistry, etc. These will be addressed in future papers. 


\section{Experimental Section}

Chemicals: The DHBC, poly(ethylene oxide)- $b$-poly(acrylic acid) (PEO- $b$-PAA, MPEO = 5000 g.mol ${ }^{-1}$, MPAA $=790$ g.mol ${ }^{-1}$ ), was obtained by atom transfer radical polymerization (ATRP) of tert-butyl acrylate with $\alpha$-bromide-functionalized PEO macroinitiators ( $\mathrm{Mw}=5000$ g.mol-1), with the subsequent deprotection reaction being done under acidic conditions. ${ }^{[40]}$ Neomycin B (NM), an aminoglycoside antibiotic, was purchased from Aldrich and used without any further purification.

Synthesis of $99 \% \boldsymbol{E}$-Bis(triethoxysilyl)ethenylene: ${ }^{[40,41]}$ In an Arpurged and flame-dried Schlenk flask, $43 \mathrm{~mL}$ of triethoxyvinylsilane $(39 \mathrm{mmol})$ with Grubbs first generation catalyst (205 mg, $0.20 \mathrm{mmol}$ ) was reacted at reflux under Ar for $4 \mathrm{~h}$. The final product was then recovered by fractional distillation under reduced pressure and collected as a colourless oil at $105{ }^{\circ} \mathrm{C}(10-2$ mbar $)$ with a yield of $91 \%$. $\left.1 \mathrm{H} \mathrm{NMR} \mathrm{(400} \mathrm{MHz,} \mathrm{CDCl}_{3}\right): \delta=1.21(t, 9 \mathrm{H}), 3.81(q$, $6 \mathrm{H}), 6.64$ (E-isomer $99 \% ; s, 1 \mathrm{H}), 6.75$ (Z-isomer $1 \% ; s, 1 \mathrm{H})$.

PICPMO Preparation: The organosilica precursor, BTEB, BTSEENE or BTSE (1.47 mmol), was added to a mixture of DHBC $(150 \mathrm{mg}, 25.9 \mu \mathrm{mol})$ with an equivalent PAA content of $0.29 \mathrm{mmol}$ and $\mathrm{NM}(177 \mathrm{mg}, 0.29 \mathrm{mmol})$ in $6.6 \mathrm{~mL}$ of a solution of $\mathrm{H}_{2} \mathrm{O}: \mathrm{PrOH}(80: 20 \mathrm{v}: \mathrm{v})$ whose $\mathrm{pH}$ was first adjusted to about 2 by addition of a $3 \mathrm{M}$ aqueous $\mathrm{HCl}$ solution. These conditions promote hydrolysis of the organosilanes without formation of the PIC micelles. After completion of organosilane hydrolysis (one hour at $30{ }^{\circ} \mathrm{C}$, with magnetic stirring at $750 \mathrm{rpm}$ ), the $\mathrm{pH}$ of the solution was increased to 6.5 by addition of a small quantity of a $3 \mathrm{M} \mathrm{NaOH}$ solution. This induces simultaneously DHBC-NM electrostatic complexation and polycondensation of the organosilane species. A precipitate was observed immediately upon increasing the $\mathrm{pH}$. The medium was left whilst stirring $\left(250 \mathrm{~min}^{-1}\right)$ for $24 \mathrm{~h}$ at $30{ }^{\circ} \mathrm{C}$. Finally, the resulting precipitate was recovered by centrifugation after several washings with water and dried at $40{ }^{\circ} \mathrm{C}$ for $48 \mathrm{~h}$, yielding a white powder.

Removal of the Polyamine: The general acid-washing procedure involved addition of the dried PICPMO material $(50 \mathrm{mg})$ to an aqueous $\mathrm{NaCl}$ solution $(5 \mathrm{~mL}, 1 \mathrm{M})$ at $\mathrm{pH} 2$, adjusted with a $\mathrm{HCl}(3 \mathrm{M})$ solution, at $25{ }^{\circ} \mathrm{C}$ to destabilize the PIC complex. After $4 \mathrm{~h}$ whilst stirring at 250 $\mathrm{min}^{-1}$, the resulting solid was collected by centrifugation $\left(10000 \mathrm{~min}^{-1}, 10 \mathrm{~min}\right)$ and washed thoroughly with water. Finally, the product was dried in air at $40^{\circ} \mathrm{C}$ for $48 \mathrm{~h}$.

Bromination: $40 \mathrm{mg}$ of BTSEENE-based PMO was introduced in a small uncapped vial, which was itself placed in a $250 \mathrm{~mL}$ beaker. Several drops of liquid bromine were added to the beaker, without direct contact with the nanoparticles. The beaker was covered with several layers of parafilm to contain the $\mathrm{Br}_{2}$ vapor and the sample was exposed to $\mathrm{Br}_{2}$ vapor for a period of $24 \mathrm{~h}$. Then the parafilm was removed and the physisorbed bromine was removed by several washings with dichloromethane, water and $\mathrm{EtOH}$. The excess bromine was neutralized with a saturated sodium thiosulfate solution. After several washings with dichloromethane and EtOH, the final product was centrifuged and dried under vacuum to be finally recovered as a pale orange powder. The pale orange coloration, instead of the expected white material, suggests that a small quantity of physisorbed $\mathrm{Br}_{2}$ remained associated with the material even after extensive washing.

\section{Acknowledgments}

The authors acknowledge Dr. Philippe Gaveau and Emmanuel Fernandez for solid state NMR experiments and Dr. Erwan Olivero for HRTEM studies (Institut Charles Gerhardt Montpellier, 
France). The authors thank Patrick Lacroix-Desmazes (ICGM Montpellier) for his assistance in the DHBC polymer synthesis. The authors thank the French Agence Nationale de la Recherche for funding of the MESOPIC Project (2015-2019), No. ANR-15-CE07-0005.

Keywords: Mesoporous hybrid organosilica $\cdot$ Micelles $\cdot$ Sol-gel processes $\cdot$ Periodic mesoporous organosilica $\cdot$ Bridged silsesquioxanes

[1] K. J. Shea, D. A. Loy, O. W. Webster, Chem. Mater. 1989, 1, 572.

[2] R. J. P. Corriu, J. J. E. Moreau, P. Thepot, M. Wong Chi Man, Chem. Mater. 1992, 4, 1217.

[3] M. Ferre, R. Pleixats, M. Wong Chi Man, X. Cattoen, Green Chem. 2016, 18, 881.

[4] V. T. Freitas, L. Fu, A. M. Cojocariu, X. Cattoen, J. R. Bartlett, R. Le Parc, J.- L. Bantignies, M. Wong Chi Man, P. S. Andre, R. A. S. Ferreira, L. D. Carlos, ACS Appl. Mater. Interfaces 2015, 7, 8770.

[5] A. Noureddine, M. Gary-Bobo, L. Lichon, M. Garcia, J. I. Zink, M. Wong Chi Man, X. Cattoen, Chem. Eur. J. 2016, 22, 9624.

[6] Y. Tsuneo, S. Toshio, K. Kazuyuki, K. Chuzo, Bull. Chem. Soc. Jpn. 1990, 63, 988.

[7] C. T. Kresge, M. E. Leonowicz, W. J. Roth, J. C. Vartuli, J. S. Beck, Nature 1992, 359, 710.

[8] Q. Huo, D. I. Margolese, U. Ciesla, P. Feng, T. E. Gier, P. Sieger, R. Leon, P. M. Petroff, F. Schuth, G. D. Stucky, Nature 1994, 368, 317.

[9] M. H. Lim, C. F. Blanford, A. Stein, J. Am. Chem. Soc. 1997, 119, 4090.

[10] T. Asefa, M. J. MacLachlan, N. Coombs, G. A. Ozin, Nature 1999, 402, 867.

[11] S. Inagaki, S. Guan, Y. Fukushima, T. Ohsuna, O. Terasaki, J. Am. Chem. Soc. 1999, 121, 9611.

[12] B. J. Melde, B. T. Holland, C. F. Blanford, A. Stein, Chem. Mater. 1999, 11, 3302.

[13] R. J. Corriu, A. Mehdi, C. Reye, C. Thieuleux, Chem. Commun. 2003, 1564.

[14] J. G. Croissant, X. Cattoen, M. Wong Chi Man, J.-O. Durand, N. M. Khashab, Nanoscale 2015, 7, 20318.

[15] A. Sayari, S. Hamoudi, Chem. Mater. 2001, 13, 3151.

[16] B. Hatton, K. Landskron, W. Whitnall, D. Perovic, G. A. Ozin, Acc. Chem. Res. 2005, 38, 305.

[17] F. Hoffmann, M. Cornelius, J. Morell, M. Froba, Angew. Chem. Int. Ed. 2006, 45, 3216; Angew. Chem. 2006, $118,3290$.

[18] N. Mizoshita, T. Tani, S. Inagaki, Chem. Soc. Rev. 2011, 40, 789.

[19] A. Noureddine, L. Lichon, M. Maynadier, M. Garcia, M. Gary-Bobo, J. I. Zink, X. Cattoen, M. Wong Chi Man, Nanoscale 2015, 7, 11444.

[20] S. Inagaki, S. Guan, T. Ohsuna, O. Terasaki, Nature 2002, 416, 304.

[21] X. Y. Bao, X. S. Zhao, X. Li, P. A. Chia, J. Li, J. Phys. Chem. B 2004, 108, 4684.

[22] C. Vercaemst, M. Ide, P. V. Wiper, J. T. A. Jones, Y. Z. Khimyak, F. Verpoort, P. Van Der Voort, Chem. Mater. 2009, 21, 5792.

[23] Y. Hu, K. Qian, P. Yuan, Y. Wang, C. Yu, Mater. Lett. 2011, 65, 21.

[24] Ref [21].

[25] C. Vercaemst, P. E. de Jongh, J. D. Meeldijk, B. Goderis, F. Verpoort, P. Van Der Voort, Chem. Commun. 2009, 4052.

[26] O. Muth, C. Schellbach, M. Froba, Chem. Commun. 2001, 2032.

[27] Y. Goto, S. Inagaki, Chem. Commun. 2002, 2410.

[28] N. Baccile, J. Reboul, B. Blanc, B. Coq, P. Lacroix-Desmazes, M. In, C. Gerardin, Angew. Chem. Int. Ed. 2008, 47, 8433; Angew. Chem. 2008, 120, 8561.

[29] D. Houssein, J. Warnant, E. Molina, T. Cacciaguerra, C. Gerardin, N. Marcotte, Microporous Mesoporous Mater. 2017, 239, 244.

[30] E. Molina, J. Warnant, M. Mathonnat, M. Bathfield, M. In, D. Laurencin, C. Jerome, P. Lacroix-Desmazes, N. Marcotte, C. Gerardin, Langmuir 2015, 31, 12839.

[31] A. Birault, E. Molina, C. Carcel, J. Bartlett, N. Marcotte, G. Toquer, P. Lacroix- Desmazes, C. Gerardin, M. Wong Chi Man, J. Sol-Gel Sci. Technol. 2019, 89(1), 189-195.

[32] A. Birault, E. Molina, G. Toquer, P. Lacroix-Desmazes, N. Marcotte, C. Carcel, M. Katouli, J. R. Bartlett, C. Gerardin, M. Wong Chi Man, ACS Appl. Biol. Mater. 2018, 1, 1787.

[33] J. Jeromenok, J. Weber, Langmuir 2013, 29, 12982.

[34] X. Li, G. Chen, J. Ma, Q. Jia, Sep. Purif. Technol. 2019, 210, 995.

[35] W. F. Giauque, J. O. Clayton, J. Am. Chem. Soc. 1933, 55, 4875.

[36] M. P. Edejer, G. Thodos, J. Chem. Eng. Data 1967, 12, 206.

[37] M. T. J. Keene, R. D. M. Gougeon, R. Denoyel, R. K. Harris, J. Rouquerol, P. L. Llewellyn, J. Mater. Chem. 1999, 9, 2843.

[38] A. Boutin, B. Coasne, A. H. Fuchs, A. Galarneau, F. Di Renzo, Langmuir 2012, 28, 9526. 
[39] M. C. Burleigh, S. Jayasundera, C. W. Thomas, M. S. Spector, M. A. Markowitz, B. P. Gaber, Colloid Polym. Sci. 2004, 282, 728.

[40] Y. Xia, R. Mokaya, Microporous Mesoporous Mater. 2005, 86, 231.

[41] C. Vercaemst, M. Ide, B. Allaert, N. Ledoux, F. Verpoort, P. Van Der Voort, Chem. Commun. $2007,2261$. 